\title{
Aux confins de la pensée, le monstre. Chassé-croisé entre Odilon Redon et Shary Boyle
}

\author{
Fabienne Claire Caland \\ Université du Québec à Montréal
}

Imaginer de nouveaux monstres en pleine expansion du naturalisme, au moment où Courbet déchaîne les passions, tourner le dos aux difformités de la nature (que le public aurait $\mathrm{pu}$ accepter plus volontiers qu'une araignée souriante) pour préférer les chimériques curiosités que son esprit lui confie, voilà qui, chez Odilon Redon, est une gageure. Qu'importe, comme il le signale dans son journal, À soi-même: "Je ne comprends rien à ce que l'on appelle des "concessions"; on ne fait pas l'art qu'on veut.» (p.23). Imaginer des monstres n'est cependant pas un travail de tout repos, comme le concède l'artiste : «j'ai subi les tourments de l'imagination et les surprises qu'elle me donnait sous le crayon; mais je les ai conduites et 
menées, ces surprises, selon des lois d'organisme d'art que je sais, que je sens, à seule fin d'obtenir chez le spectateur, par un attrait subit, toute l'évocation, tout l'attirant de l'incertain, sur les confins de la pensée» (p. 26). Sans entrer dans les détails des mécanismes de la réception, il est frappant de noter combien ce pouvoir de l'incertain, ce cheminement du spectateur vers les « confins de la pensée » s'accorde parfaitement à la démarche de l'artiste ontarienne Shary Boyle. Frappant, aussi, de saisir en quoi, par des techniques et des médiums différents, chacun des deux artistes euphémise le monstre. Aux confins de la pensée, celui-ci offre une image presque apaisée, de l'ordre de cette inquiétante étrangeté freudienne qui a fait les beaux jours de la pensée occidentale de la première moitié du $20^{\text {e }}$ siècle.

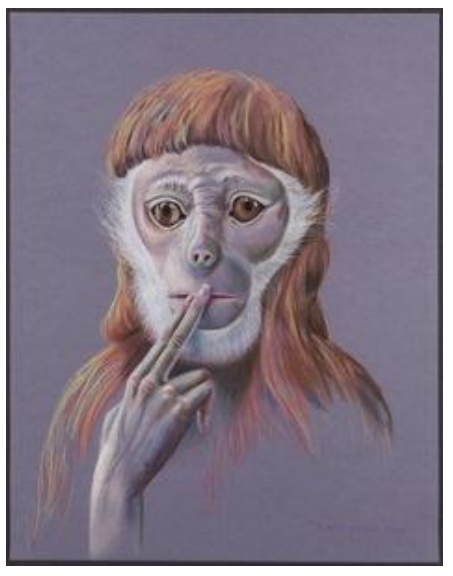

\section{Les singeries du monstre}

«À côté d'une incertitude, mettez une certitude. ${ }^{1} »$

S'il faut partir du connu pour fabriquer du monstre, l'homme est un choix de gourmet. Ainsi en a décidé la physiognomonie animale, dont on repère la complexité dans Our Ancestor's Concern (L'inquiétude de notre ancêtre, 2009), un pastel de Shary Boyle: un visage de singe dont la main de femme est posée sur la bouche pour la réduire au silence.

${ }^{1}$ Propos tenus par Corot à Redon (Redon, p. 36). 
Que veut-elle taire ? Sa monstruosité animale ? Elle ferait alors, par un mutisme conscient, un pied de nez aux animauxmachines de Descartes, auxquels sont refusées conscience et pensée : «Et on ne doit pas confondre les paroles avec les mouvements naturels qui témoignent les passions et peuvent être imités par des machines aussi bien que par les animaux ; ni penser, comme quelques anciens, que les bêtes parlent, bien que nous n'entendions pas leur langage. » $(1637$, p. 68)

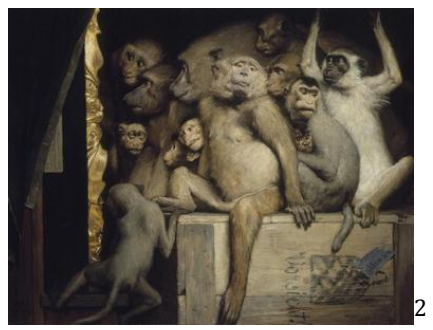

Our Ancestor's Concern fait écho à l'insolite Portrait d'Antonietta Gonsalus, peint vers 1594-1595 par Lavinia Fontana, où le modèle féminin fixe le spectateur droit dans les yeux, lui sourit avec douceur, alors qu'il est happé par la dissonance entre son visage entièrement couvert de poils et ses mains blanches et glabres, dissonance encore plus saisissante du fait de la richesse de ses vêtements.

\footnotetext{
2 Cette théorie machiniste repose sur l'idée que les corps humains et animaux sont des machines mais que, dans le cas de l'homme, il y a en plus une âme. Cette position sera largement discutée par son implication: les animaux n'auraient pas d'âme. Darwin, par exemple, s'oppose à cette différenciation de nature. Pour lui, ce qui sépare l'homme de l'animal est de l'ordre du degré. On pourra, dans le domaine de l'art, se référer à l'œuvre du peintre autrichien Gabriel von Max (1840-1915). Ce darwiniste a élevé un troupeau de singes qui vont lui servir de modèles. Entre autres, on pourra voir Jury of Apes (ou Monkeys as Critics, Monkeys as Judges of Art), 1889.
} 


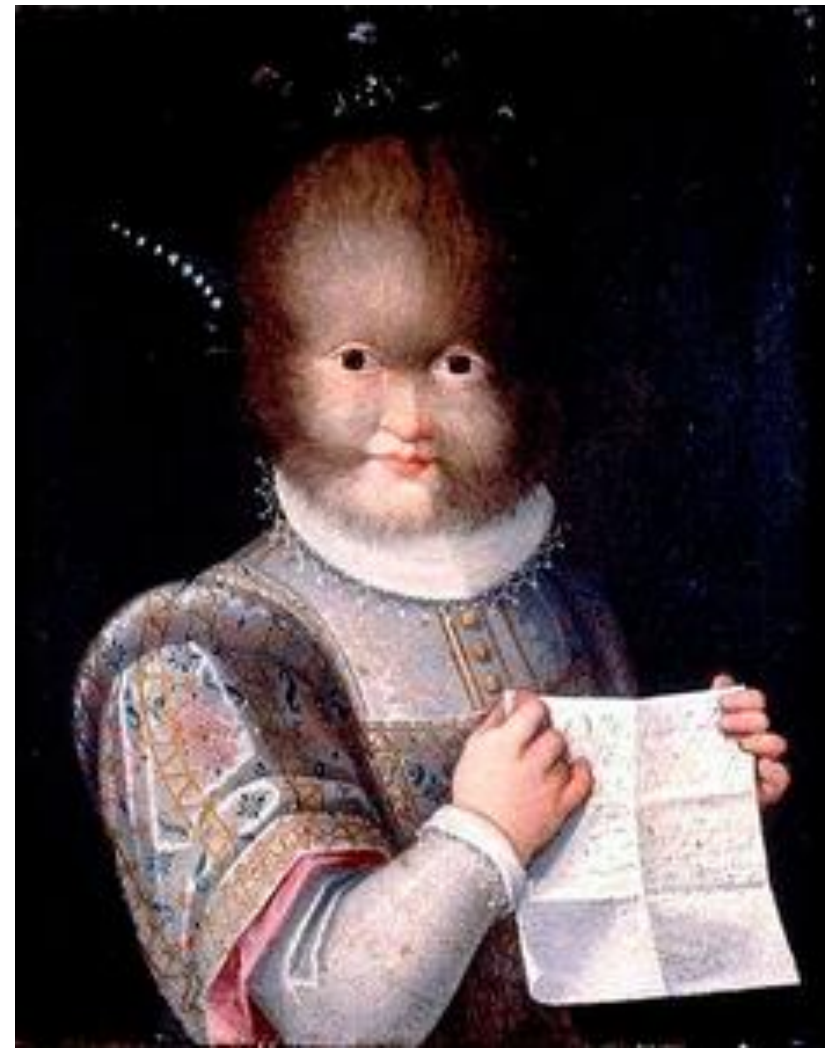

Portrait d'Antonietta Gonsalus de Lavinia Fontana, vers 1594-1595

Même mécanisme symbolique, donc, dans les deux cas : le spectateur est face à la monstruosité d'un hybride dont la juxtaposition de l'humain et de l'animal le gêne. Il ressent une forme de violence malaisée et soutenir le regard du modèle, que ce soit chez Fontana ou chez Boyle, est difficile. La différence fondamentale entre les deux existe hors du cadre du tableau, somme toute. Atteinte d'hypertrichose, Antonietta Gonsalus, appelée aussi Tognina, a existé et fit même merveille à la cour de Marguerite d'Autriche. Sur le tableau, elle tient entre ses 
mains délicates d'enfant un papier officiel qui prouve son origine, expliquant qu'elle est la fille de Petrus Gonsalus, venu des îles Canaries. Quant aux mains du singe-femme de Boyle, elles ne tiennent rien, et pour cause : l'existence du monstre n'est redevable qu'à l'imagination fertile de son artiste.

Sortie des confins de la pensée, la femme-singe n'est pas nommée. Elle n'a pour autre origine que l'inspiration d'une tradition de physiognomonie remontant à l'Antiquité mais qui, dans notre cas, semble essentiellement tributaire du bouleversement qu'a apporté l'étude des affections que subit l'âme par l'action du corps ${ }^{3}$ (Les passions de l'âme de Descartes date de 1649) sur le travail novateur d'un Charles Le Brun. Autrement dit, l'association syllogistique (chaque espèce animale a des traits particuliers ; ces éléments se trouvent aussi chez l'homme; l'homme possédant les mêmes traits qu'une espèce particulière a donc le même caractère qu'elle ${ }^{4}$ ) se tempère du moment qu'à son désir de permanence, la passion au sens cartésien oppose l'éphémère, le passager. Tout en composant des portraits d'animaux expressifs où se lit une intelligence d'humain, Le Brun met d'ailleurs l'accent sur la forme des sourcils et des yeux, qui trahissent si bien les

3 On saisit alors à quel point, dans la conception de Descartes, la passion (essentielle à la survie humaine parce qu'elle nous connecte au monde) est passive. Elle renvoie aux états affectifs que nous connaissons sous le nom d'émotion, de plaisir, de douleur. En conséquence, chacun l'éprouve.

${ }^{4}$ Ce syllogisme se retrouve au sein de nombreux travaux. José Joaquim de Gama Machado (1831), par exemple, affirme qu'au sein des sciences humaines « se trouvent des formes, des robes et des couleurs identiques, dans l'immense série des êtres organisés » et "se rencontrent aussi les mêmes conformités d'instincts, d'habitudes et de mœurs ». En conséquence, affirme-til, cette «loi est particulièrement applicable au règne animal ; car toutes les fois qu'un être offre avec quelque autre, dans les diverses parties dont il est composé, des ressemblances extérieures, son caractère moral doit tenir à la fois de celui de chacun des animaux dont il se rapproche ». 
émotions, les passions. Pourvoit-il le lion et le cheval d'yeux humains que Boyle adopte le même procédé sur un visage simiesque.

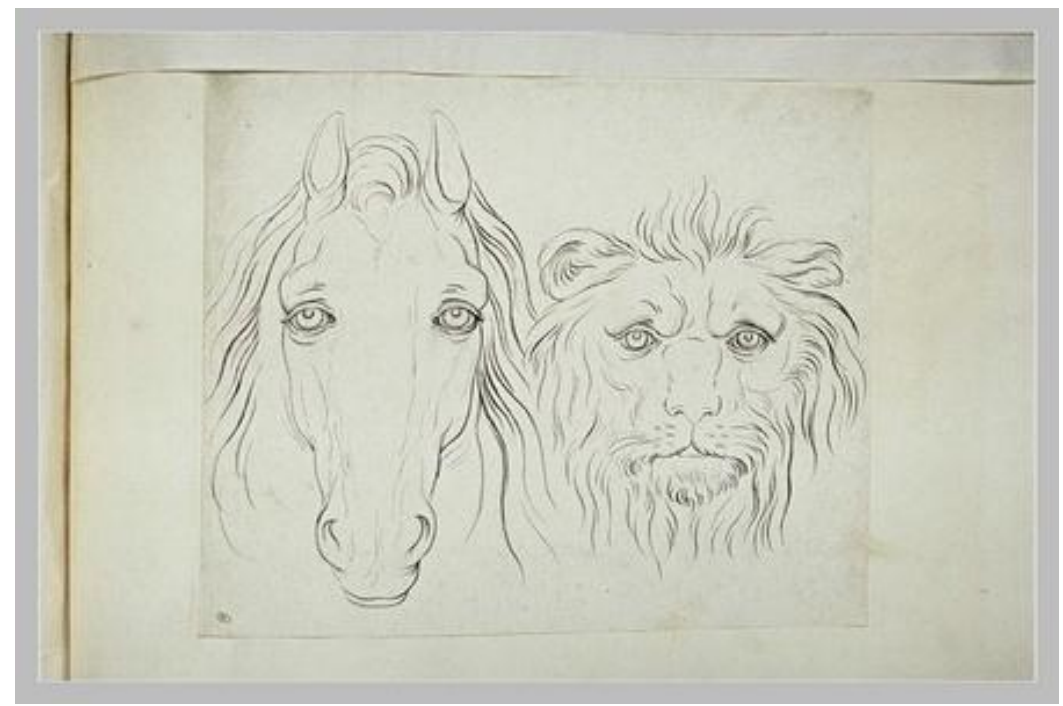

Une tête de cheval et une tête de lion, Charles Lebrun, $17^{\mathrm{e}}$ siècle

Le voilà donc ce singe-là, cet ancêtre gratifié non pas d'un regard malin ou concentré comme dans les études de Le Brun ${ }^{5}$ mais triste, presque humide de larmes d'homme (ou de femme, en l'occurrence). Affligé, le singe-femme captive le spectateur au point de le faire pénétrer en dedans de ce masque animal, dans un univers confus dont le seul indice (qui n'en est pas un !) est que le silence fait loi. Tristesse de ne pouvoir parler ou mutisme volontaire de tristesse, nous n'en saurons guère

5 Je pense ici à son étude Quatre têtes d'hommes en relation avec le singe et Deux têtes de singes, l'une de profil et l'autre de face (Paris, Musée du Louvre, département des Arts graphiques). 
davantage que ce que le titre nous livre, pudiquement: Our Ancestor's Concern.

Quelle drôle de singerie en somme que celle de Boyle! Nous sommes à la fois proche de ce courant ornemental qui accompagna les célèbres chinoiseries et les oubliées papillonneries du $18^{\mathrm{e}}$ siècle, par sa visible volonté d'esthétisation, et si loin de son intention première, dans la lignée des artistes qui ont préféré s'éloigner d'une charge souvent moqueuse envers les primates pour préférer une certaine gravité, comme Alexandre Gabriel Decamps au $19^{\mathrm{e}}$ siècle.

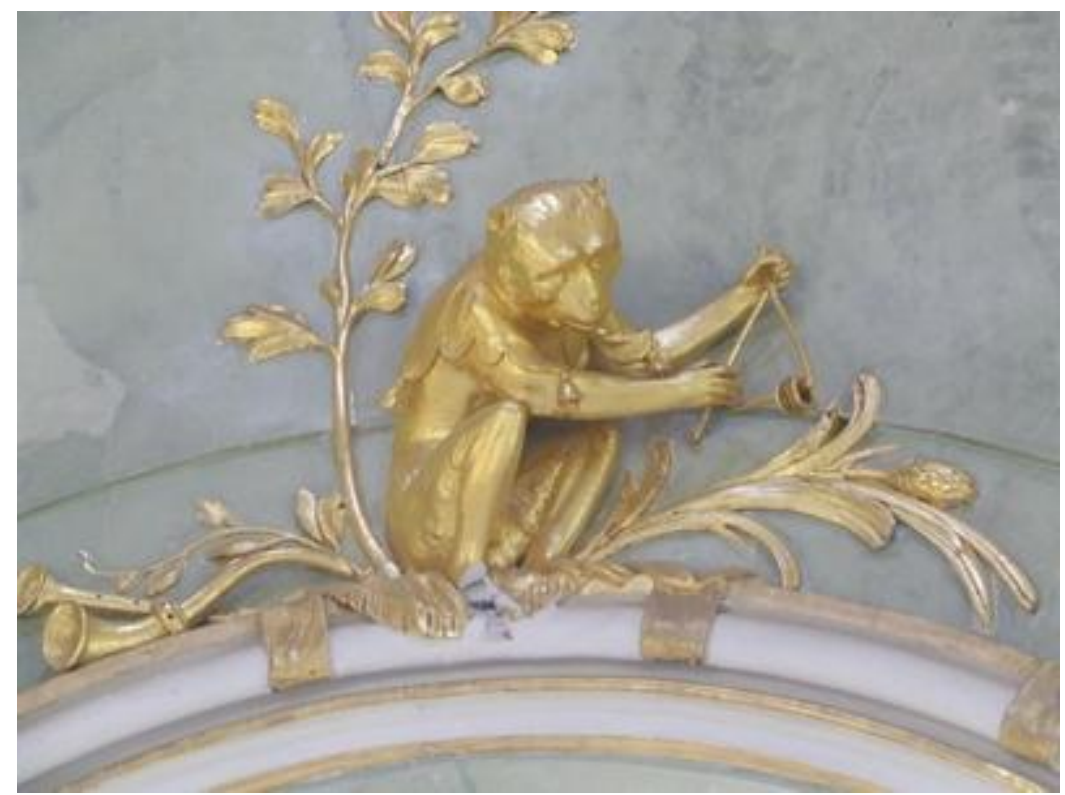

Détail d'un plafond du pavillon chinois dans le parc du Palais de SansSouci à Potsdam, $18^{\mathrm{e}}$ siècle 


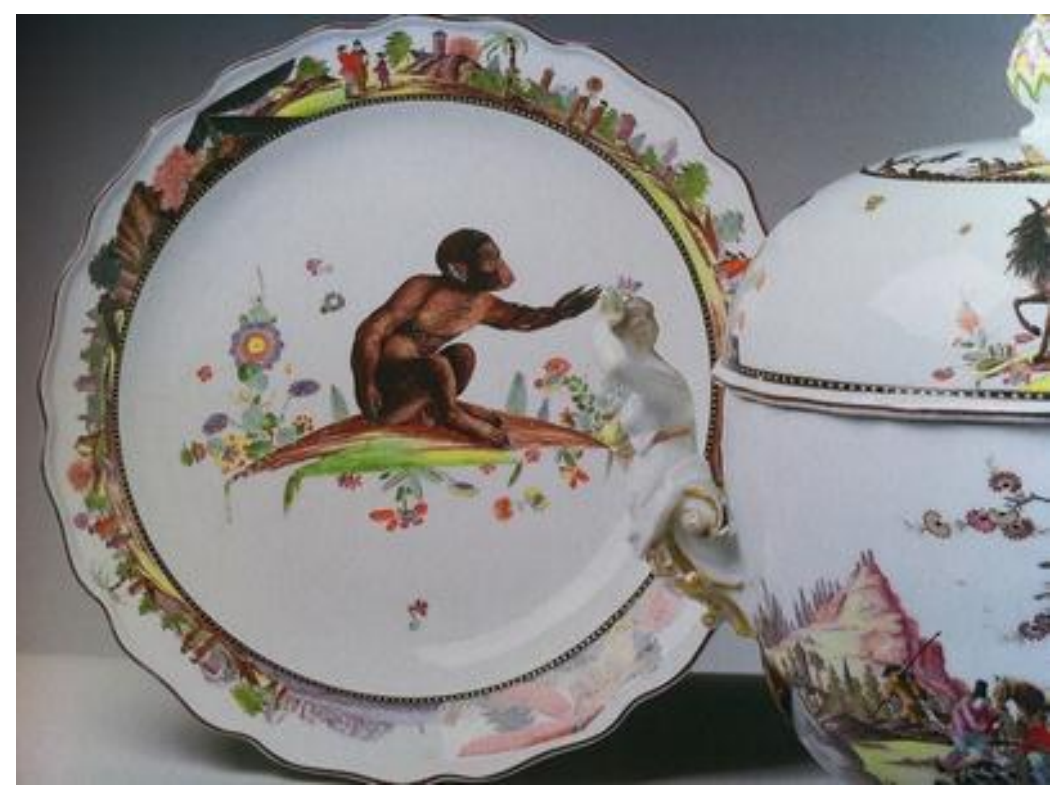

Assiette d'inspiration orientale, Messein, 1735-1740

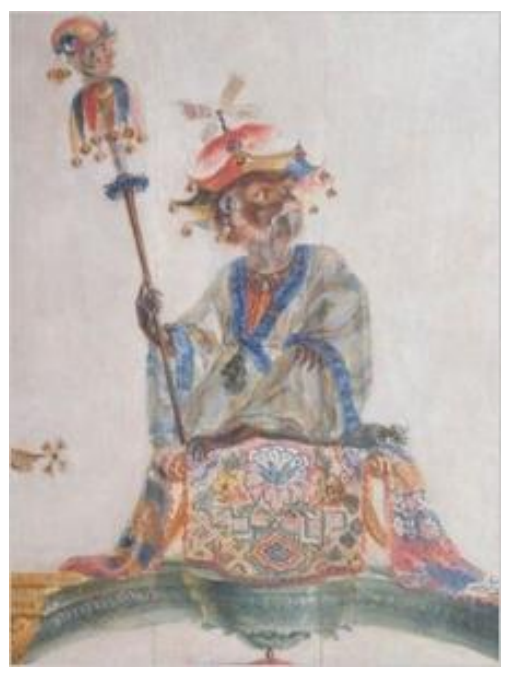

Détail de la Grande Singerie du château de Chantilly, France, $18^{\mathrm{e}}$ siècle 
Que l'on songe à son Singe peintre, dit aussi Intérieur d'atelier' : sous les traits de l'animal transperce l'état d'âme d'un peintre qui s'adonne à son art sans autre visée que de transcrire la réalité.

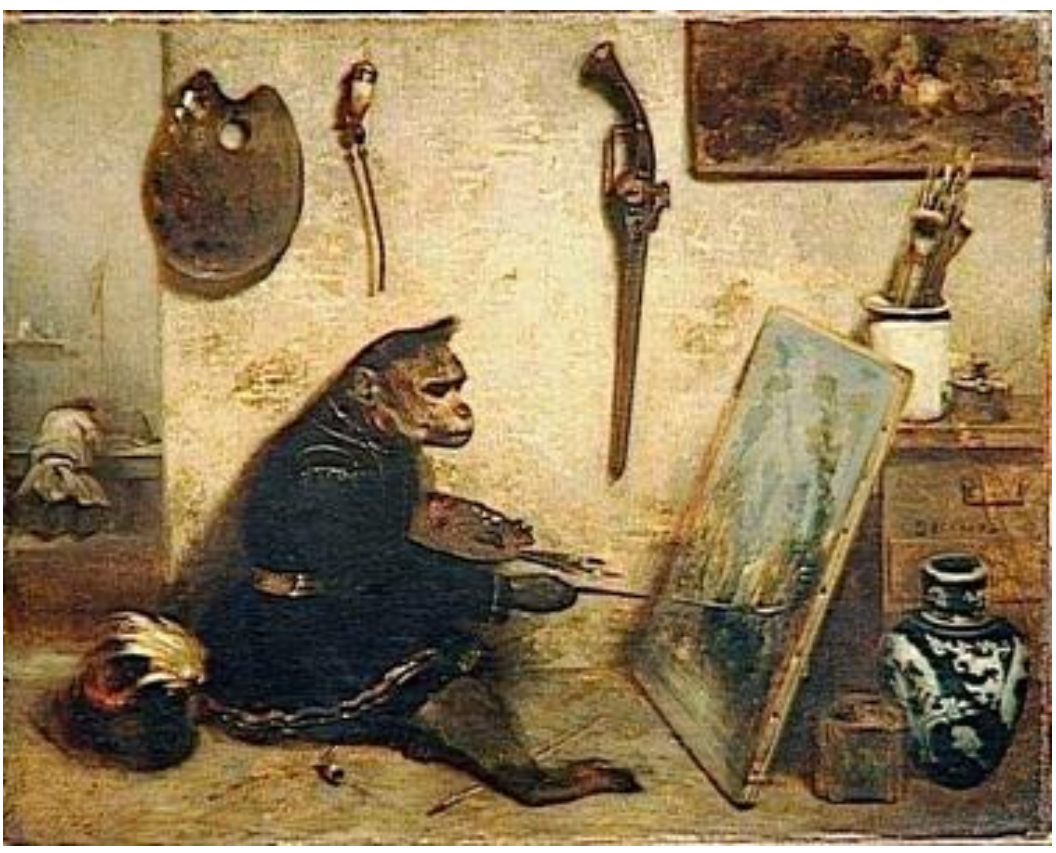

Singe peintre, Alexandre Gabriel Decamps, vers 1833

" On ne fait pas l'art qu'on veut », écrit Redon, manière au contraire de dire que l'art doit être transfiguration et non imitation. Certes, et Boyle après lui pourrait faire sienne cette affirmation: «On ne peut m'enlever le mérite de donner

6 Composé vers 1833, ce tableau est exposé au Louvre. Il a pour inscription : "Cette "singerie" s'inscrit dans une tradition iconographique héritée de Teniers et illustrée en France au siècle précédent par Chardin. Decamps porte un regard ironique sur son art. Le peintre, qui imite au lieu d'inventer, est caricaturé en "singe savant", portant le costume d'écuyer des animaux de cirque. » 
l'illusion de la vie à mes créations les plus irréelles. Toute mon originalité consiste donc à faire vivre humainement des êtres invraisemblables selon les lois du vraisemblable, en mettant, autant que possible, la logique du visible au service de l'invisible» (Redon, p. 28). Dans ce travail, le médium est fondamental: le pastel et la porcelaine pour Shary Boyle, le fusain pour Redon. À médiums différents, résultats identiques : l'impression d'une réalité autre, favorisée par le choix technique qui, quand il s'agit de rendre compte du monstre, en atténue la charge violente.

\section{Au service de l'apaisement monstrueux : le fusain et le pastel}

Our Ancestor's Concern questionne l'évolution darwinienne, l'accès au langage comme preuve d'humanité, le travail de création de l'artiste et aussi - ce qui est essentiel chez l'artiste — la féminité dangereuse. Le choix du pastel n'a rien d'anodin ; il oriente la lecture aussi sûrement que le fusain cher à Redon pour composer les monstres qui lui sont chers, de l'araignée au polype difforme en passant par l'homme-cactus.

Le pastel est parfait pour le rendu des tonalités de la chair. De fait, sa technique est souvent adoptée pour les nus et les portraits, alors qu'elle est délaissée pour les paysages. En effet, le pastel permet le rendu du flou, d'effets vaporeux que les paysagistes, hormis les Impressionnistes, ne recherchent pas. Quand Redon utilise le pastel ${ }^{7}$, quand il se met tardivement à la couleur après un long passage en noir et blanc - parce que l'essentiel pour lui est devenu plus la lumière que l'ombre, et ce, en accord avec un cheminement mystique - , il le fait pour

\footnotetext{
7 Redon adopte aussi une technique mixte : fusain et pastel, pastel et craie ou pastel et peinture à l'huile.
} 
mieux saisir la carnation des visages (Le portrait de Madame Redon, 1880) et des corps (La naissance de Vénus, 1912). Disonsle aussi: le pastel, que trahit à chaque fois l'épreuve de la reproduction, a un je-ne-sais-quoi de gourmand dans sa texture grumeleuse et humide. Comment dans ce cas Our Ancestor's Concern pourrait-il susciter un rejet alors que sa technique même nous invite à presque le goûter ? Grâce au pastel, Boyle élimine la cruauté de son sujet, apaise la violence de cette mutation étrange dont les yeux trahissent l'âme. Elle l'humanise, l'euphémise : avec elle, le monstre est une étape transitoire sur le long chemin de l'évolution, non pas un état permanent.

Redon arrive au même résultat (élimination des tensions, apaisement de la violence) par le choix du clair-obscur pour ses dessins qui découlent « de la vision du monde mystérieux des ombres, à qui Rembrandt, en nous le révélant, donna le Verbe » (Redon, p. 28). Cette reconnaissance avouée, avec sa dette à Vinci pour son sfumato, il ne reste plus à l'artiste qu'à nous prendre à partie : «Mais je vous le dis aujourd'hui, en toute maturité de conscience, et j'y insiste, tout mon art est limité aux seules ressources du clair-obscur et il doit aussi beaucoup aux effets de la ligne abstraite, cet agent de source profonde, agissant directement sur l'esprit. » (Redon, p. 25) Pour obtenir ce sfumato dès 1875, l'artiste a dédaigné la peinture pour le fusain, en plus de la mine de plomb : il parviendra d'ailleurs à une telle maîtrise qu'avec lui, le fusain acquiert une noblesse qu'on lui refusait jusque là. Accusé d'être "plaisant», Redon démontrera combien, au contraire, il est « grave $»^{8}$.

8 «C'est une matière mal vue par des artistes, et négligée. Que je le dise pourtant, le fusain ne permet pas d'être plaisant, il est grave. » (Redon, p. 25) 
Face à son Araignée qui pleure (1881), la monstrueuse hybridité s'efface devant le chagrin visible de cette tête humanisée, devant ces joues bouffies de larmes.

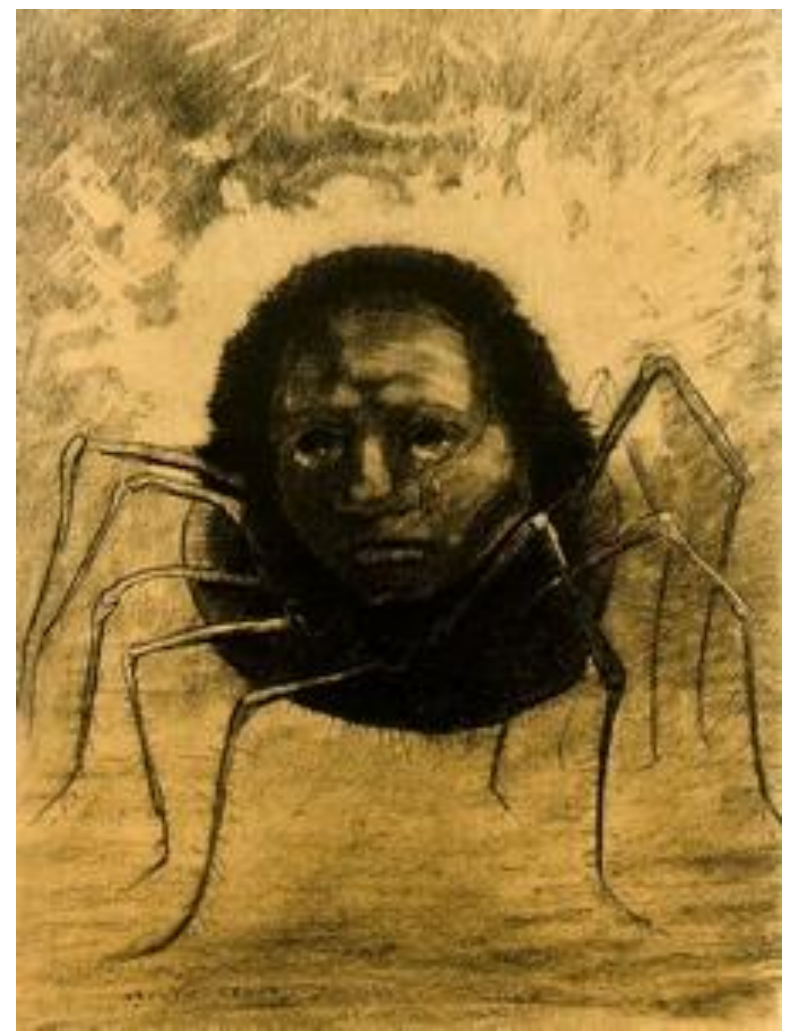

L'araignée qui pleure, Odilon Redon, 1881

Elle est peu comparable à son célèbre pendant, L'araignée qui sourit, fusain lithographié en 1887 : la première accentue nettement l'animalité (les pattes de l'araignée sont poilues) et de l'humanité (la tête humaine, aux traits masculins) quand la seconde joue à l'inverse avec des pattes fines comme des jambes et une tête entièrement poilue. 
F. C. CALAND, « Chassé-croisé entre Odilon Redon et Shary Boyle »

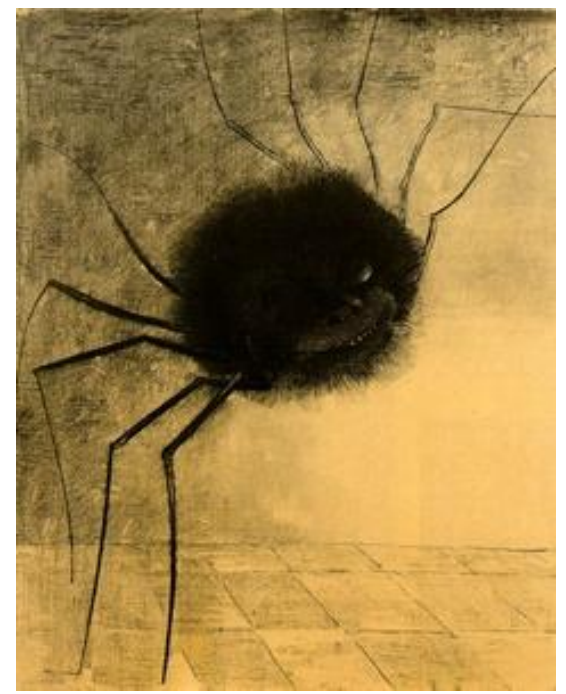

L'araignée qui sourit, Odilon Redon, 1881

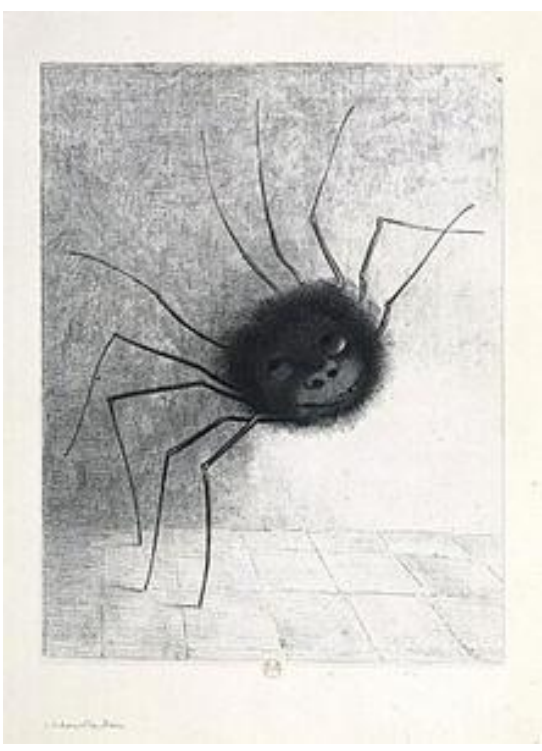

L'araignée qui sourit, Odilon Redon, 1887 (lithographie) 
Le sourire de cette dernière n'en est que plus cocasse, amusant et pas un instant effrayant ou déstabilisant. Ce monstre-là se réduit à une fantaisie équivalente à une singerie. Il en va tout autrement de L'araignée qui pleure, empreinte de cette gravité qui l'éloigne de manière significative de la fantaisie et qui procure une forme de choc plastique, une émotion esthétique au spectateur (là encore forcément déçu par les reproductions, incapables de rendre les différentes nuances du fusain, de l'aile de corbeau à l'ébène, du Dorian au Jais, en passant par des gammes de gris, de brun et de roux). Comme chez Boyle, le malaise naît de la distorsion et le médium accentue la portée symbolique de l'ensemble, à la manière d'un Rembrandt qui « a donné une vie morale à l'ombre » (Redon, p. 35). De plus, parce qu'il traduit l'ombre et brouille le modelé des formes, le fusain semble faire surgir l'araignée de la Nature elle-même qui, jusque-là, l'aurait jalousement tenue enfermée, et avec elle se sont échappées les plus incroyables créatures, dont une tête hérissée de piquants et plantée dans un pot pour le troublant fusain non daté Homme-cactus.

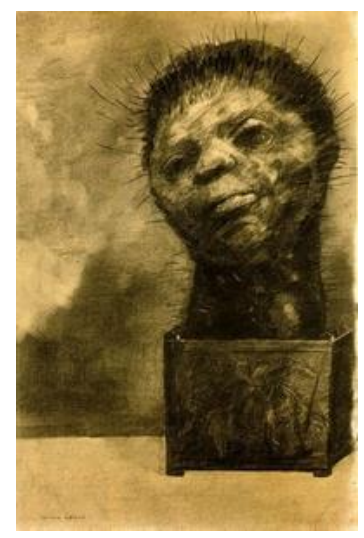

L'homme-cactus, Odilon Redon, sans date 
Chez Redon, le fusain est totalement adéquat pour traduire l'indéterminé, pour jouer de l'ombre et de la lumière, pour capter les formes plus étranges. À chaque fois transparaît son amour pour les créatures monstrueuses issues de multiples rencontres esthétiques (Vinci, Rembrandt, Gauguin) et scientifiques (Darwin, Pasteur, Lamarck, etc.). Car que ce soit L'araignée qui pleure ou L'homme-cactus, nul doute que ces monstres effondrés de douleur se chargent d'états d'âme et que c'est pour cette raison aussi qu'ils sont dessinés avec amour.

Comme les noirs angoissés de Redon, le pastel qu'utilise Boyle contribue à enclore la violence dans l'œuvre: non pas l'exclure donc, mais la circonscrire. Le monstre est apaisé, défait de sa vêture féroce, pour se situer quelque part entre l'homme et la bête. Le fusain obtient ce résultat par son effet clair-obscur, par une certaine mollesse rendant les contours diffus, enfin par sa profondeur. Le pastel, lui, y accède par l'effet de carnation et de sensualité. Mais il ne faudra pas s'y tromper : ce médium n'est pas celui que privilégie Boyle dans son œuvre, d'abord formée au dessin. Si le pastel s'adapte parfaitement au sujet d'Our Ancestor's Concern, comme un texte appelle son genre en littérature, il est somme toute peu employé dans l'ensemble de sa création artistique. C'est la porcelaine, et non le pastel, qui est à Boyle ce que le fusain est à Redon.

\section{La porcelaine anti-violence de Boyle}

Le fusain parvenait, sous les doigts de Redon, à obtenir une profonde densité. Rien de tel avec la porcelaine dure aux contours précis, à la blancheur laiteuse. Composée d'une pâte faite de kaolin, de feldspath et de quartz, impossible à rayer avec l'acier, elle connaît un renouveau important depuis 
quelques années, comme la céramique d'une manière générale, et le réinvestissement va de l'univers fantaisiste de Claire Partington aux violences politiques de Charles Krafft en passant par les modèles contemporains revisités avec ironie de Barnaby Barford, les femmes décapitées de Jessica Harrison ou encore les curieuses métamorphoses blanches de Kate MacDowell. Boyle y occupe une place de choix et, avec elle, les figurines de porcelaine trouvent une déclinaison nouvelle.

L'artiste ontarienne travaille la porcelaine en brillance plutôt qu'en transparence, s'éloignant ainsi de la grande quête européenne de translucidité menée par les Européens devant la grandeur de la porcelaine chinoise. Elle rehausse ses figurines de dorure appliquée ici et là, allège son éclat par un trait précis et des touches de couleur et lustre l'ensemble. Lors d'un séjour de formation en Allemagne, où elle apprit les méthodes employées à Meissen, siège de la création de la porcelaine européenne au début du 18 siècle, Boyle en a acquis la parfaite maîtrise dans l'esprit des grands porcelainiers de Saxe tels que l'Europe en produisit dès le $17^{\mathrm{e}}$ siècle.

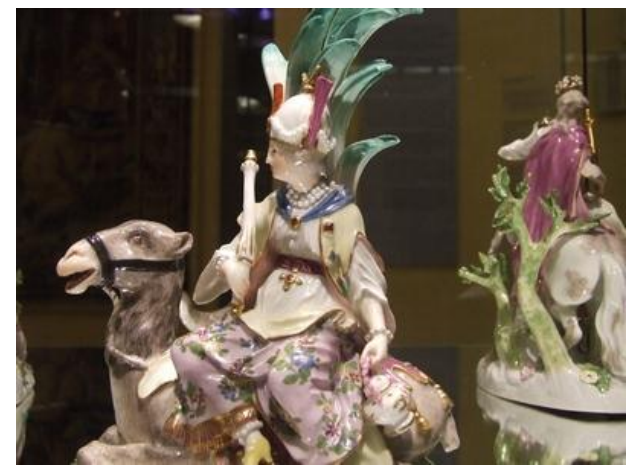

Détail de Die vier Erdteile, Johann Joachim Kaendler, Meissen, 17451746 


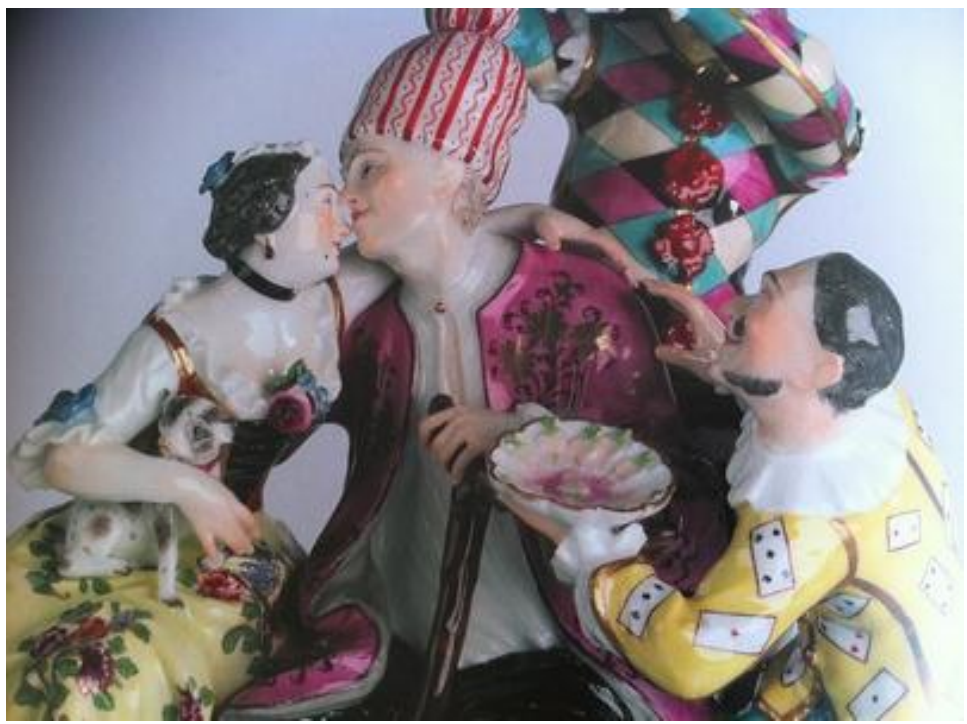

Détail de The Old Coxcomb, J. J. Kaendler, Meissen, Meissen, 1740

Forte de cet apprentissage, l'artiste avoue que ce qu'elle aime dans la porcelaine, c'est la contradiction permanente qu'elle est capable d'offrir entre force et fragilité, élégance classique et subversion. Nul doute néanmoins que la contradiction ne l'intéresse qu'à partir du moment où elle peut viser à une harmonie esthétique, prodiguer un choc visuel sans qu'une répugnance devant le sujet puisse jamais venir contrecarrer la beauté visée.

Il faut comparer certaines figurines de Jessica Harrison et de Shary Boyle pour mieux saisir l'harmonie que cherche l'Ontarienne. Dans une série de 2010, les femmes d'Harrison exhibent un cou tranché, des yeux arrachés, une cervelle mise à nu ou des entrailles sanguinolentes (dans l'ordre Mairi, Caroline, Amy Jane, Rosamund). 


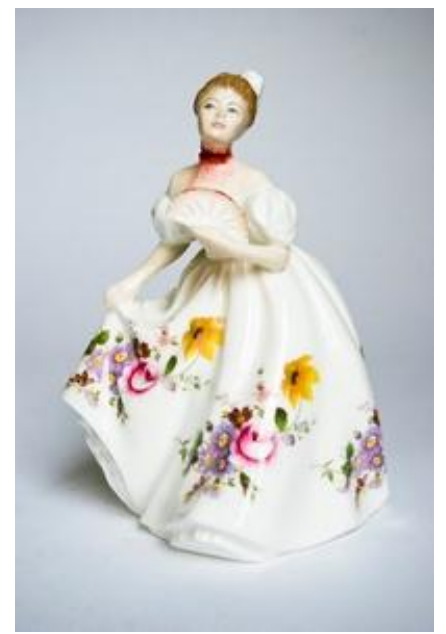

Mairi de Jessica Harrison, 2010

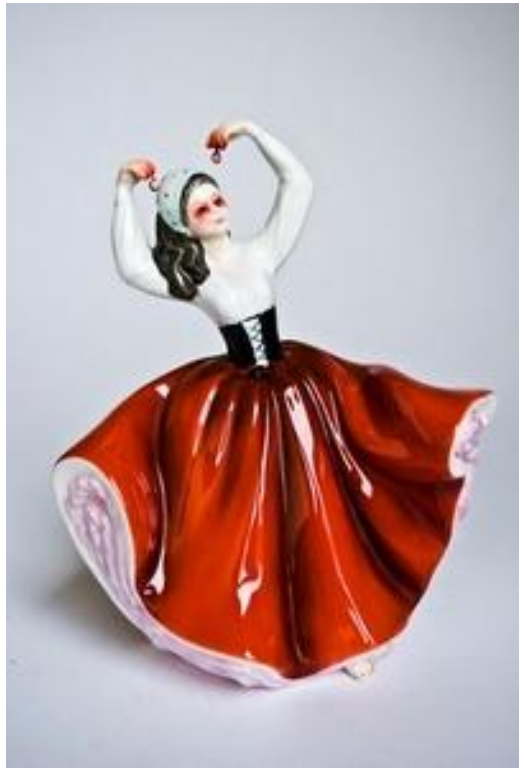

Caroline, Jessica Harrison, 2010 
F. C. CALAND, « Chassé-croisé entre Odilon Redon et Shary Boyle »

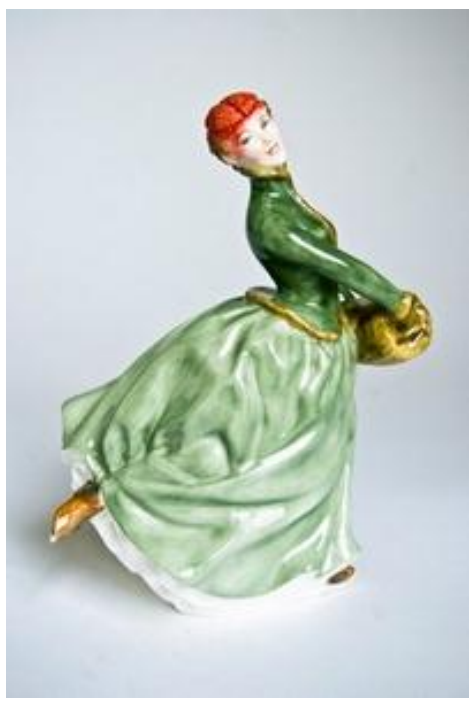

Amy Jane, Jessica Harrison, 2010

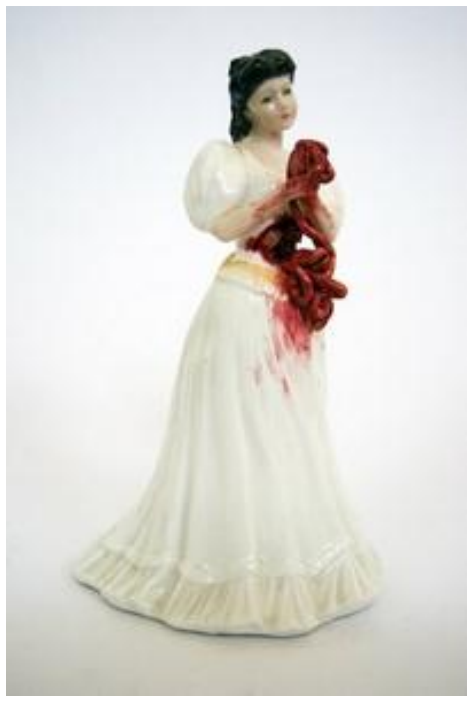

Rosamund, Jessica Harrison, 2010 
L'horreur du spectateur l'emporte sur toute considération esthétique, d'autant que ces figurines sont personnalisées par l'obtention d'un prénom et de costumes différents, d'époques différentes, etc. Elles ne sont pas interchangeables. À l'inverse, chez Boyle, elles sont anonymes. Par conséquent, leur est permis l'accès au rang de références atemporelles sorties d'un univers féérique, avec leur robe de dentelles aux allures princières. Or, puisque la violence est inhérente aux contes de fées et aux mythes, elle devient acceptable pour le regard, quoique dérangeante comme peut l'être le Portrait d'Antonietta Gonsalus. Après tout, Fontana aussi, en jouant sur le contraste entre fascination (beauté des mains, richesse du costume, grâce) et répulsion (le petit visage poilu), enferme Antonietta dans le genre du merveilleux dont elle ne peut s'extraire qu'à force d'explications contextuelles. Chez Boyle, la femme porte sa tête à bout de bras ou des mouchoirs de dentelle sur les joues (deux figurines de 2005), joue au faux fantôme et à la vraie suppliciée (Haunt, 2004) ou, enfin, tombe en arrière, décapitée (Tumbleweed, 2010). Le spectateur ne s'y trompe pas, qui enclenche immédiatement une narration merveilleuse dans laquelle ces terribles événements font sens.

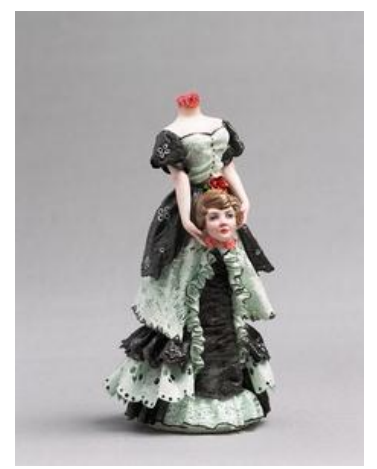

Sans titre, Shary Boyle, 2005 
F. C. CALAND, « Chassé-croisé entre Odilon Redon et Shary Boyle »

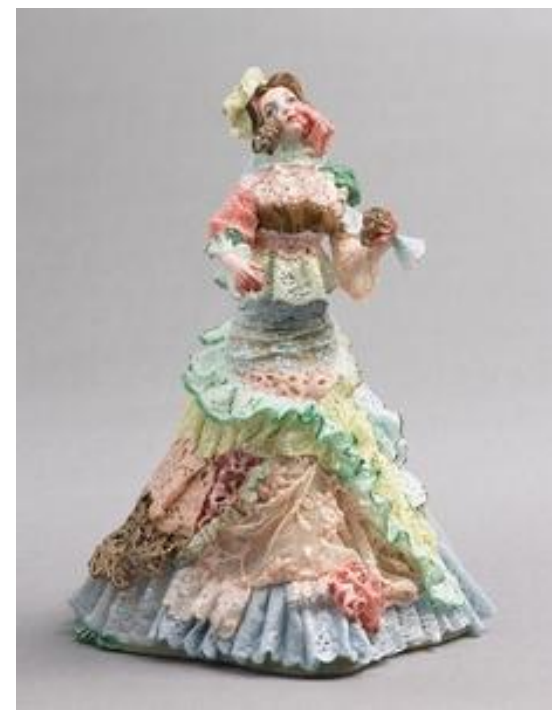

Sans titre, Shary Boyle, 2005

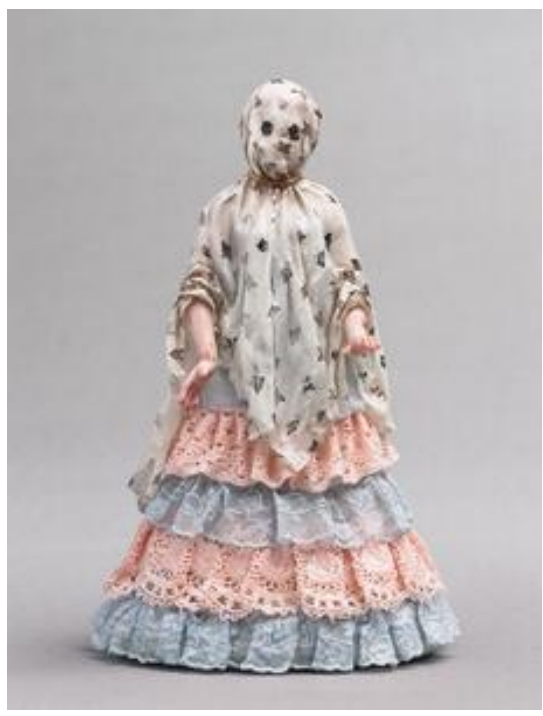

Haunt, Shary Boyle, 2004 


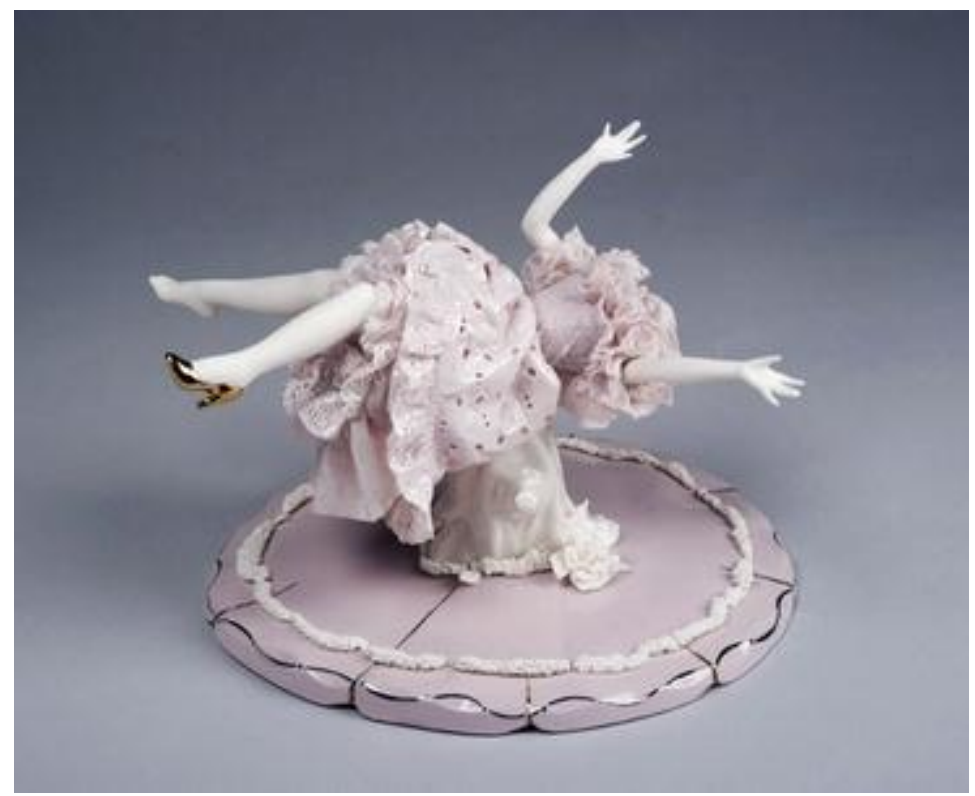

Tumbleweed de Shary Boyle, 2010 - 21Boyletumbleweed.jpg>

Une différence de taille entre Harrison et Boyle réside dans le traitement de la porcelaine et de ses couleurs: la première joue du drapé et de couleurs franches appliquées sur de grandes surfaces; la seconde emploie une gamme variée de coloris en suivant la tradition des figurines de Saxe, adopte fréquemment des teintes aux accents printaniers, affectionne les nuances pâles d'incarnadin doré, de pêche, de thé, dont la transparence sied à la porcelaine. L'artiste ajoute enfin au drapé le traitement de minutieuses dentelles dont elle se fait d'ailleurs l'une des spécialités. Une figure de dentelles? C'est Shary Boyle! Nul avant elle, dans l'art contemporain, n'a réussi à renouveler à ce point la porcelaine par une précision, une 
délicatesse de dentellière qui transforme la matière, brise son caractère inachevé, défait son aspect lisse par l'infini ajouré.

Redon use de son fusain pour créer des sfumato d'où semblent sortir des créatures monstrueuses. Boyle aère sa porcelaine. Ses créatures monstrueuses sont comme expulsées de monceaux de dentelles, engendrées par elles si l'on pense à l'incroyable Maypole (2010) d'où jambes et têtes de femmes jaillissent d'un exubérant jupon de dentelle couleur chair.

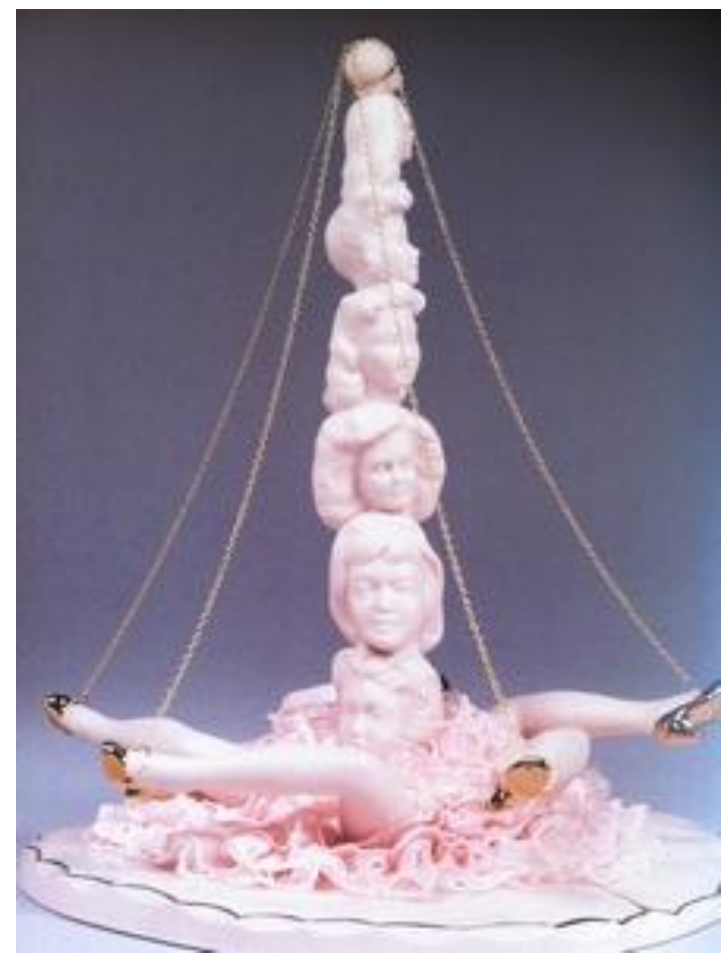

Maypole, Shary Boyle, 2010 
Elles ne naissent pas de la nature (dessinée par ces noirs qui forment une sorte de chaos organisateur chez Redon), mais d'un univers culturel illustré par le foisonnement de dentelles : d'ailleurs, dans Maypole, elles jaillissent de manière ordonnée pour créer un mât de chair féminine qui, empilée, prend la place des traditionnelles figurines représentant les corps de métier sur les arbres de mai exposés de la Bavière au Massachusetts. Pas de cordonnier, de menuisier, de charpentier ici. Être femme, un métier? Les jambes parfaitement galbées à la manière d'une pin-up ou d'une poupée Barbie sont enchaînées (avec des anneaux en or et des chaînes de métal), ce qui est bien sûr lourd de sens : le monstrueux féminin est jusqu'à aujourd'hui jugulé. Mais à bien y regarder, ce sont comme des bijoux qui ne paraissent pas le moins du monde entraver le corps. La femme serait à ce point assujettie qu'elle en aime ses chaînes? Quoiqu'en métal, celles-ci s'harmonisent parfaitement aux chaussures de porcelaine dorée. Le féminisme de Boyle s'exprime sans fards, comme dans l'ensemble de son œuvre et, à chaque fois, cette revendication est esthétisée. Elle fait de la porcelaine un matériau anti-violence.

\section{Le baiser de la femme-chauve-souris}

Qu'une femme bleue de corps et de robe enfile une chaussure dorée amuse le spectateur (Silver Buckle, 2010), lui fait croire d'abord à une coquetterie du sujet redoublée par une coquetterie ornementale frôlant le kitsch ; que, pour accomplir ce geste, elle se penche vers une troisième jambe sortie de ses jupons le rend mal à l'aise, comme face à cette inquiétante étrangeté où l'insolite se mêle au familier. 


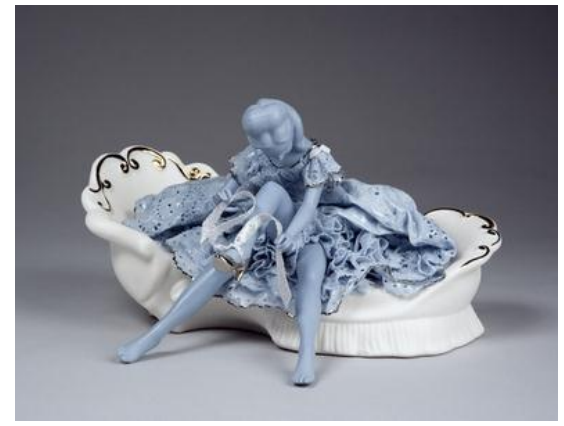

Silver Buckle, Shary Boyle, 2010

Pourtant, nous sommes, depuis un Redon précurseur des surréalistes à bien des égards à une Boyle née comme tant d'artistes de l'après-guerre avec le langage freudien au bout des lèvres, face à un évident malaise qui active une pensée psychanalytique en plus d'une pensée magique. Une très belle femme aux multiples paires d'yeux sur le front et paires de bras sortant des flancs (Untitled, 2005) renvoie alors au conte de fées par ses efforts de tisseuse (figure du destin) et son soulier posé à terre (une princesse en manque de prince) de même qu'à une féminité dangereuse sous des dehors accueillants.

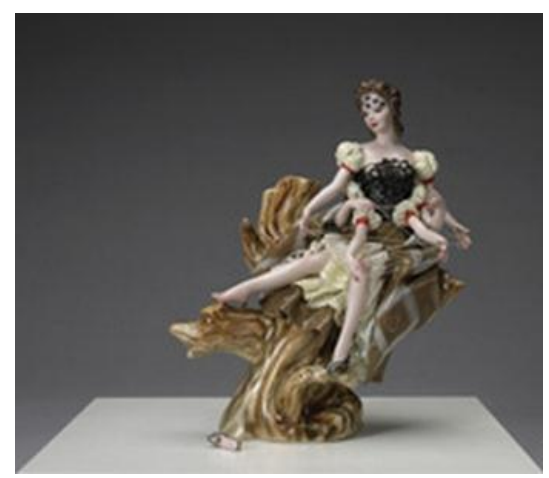

Sans titre, Shary Boyle, 2005 
D'autres sculptures posent les questions du sexe, de la castration, de manière plus crue par l'image monstrueuse à l'excès, par exemple, de la femme-phallus en porcelaine (Intersects, 2009). Mais une telle radicalité est rare, somme toute. On la trouverait ailleurs, chez sa contemporaine anglaise Annie Attridge, dont les sculptures en porcelaine semblent inspirées de Boyle mais dans une version moins élaborée et poétique9. De son côté, refusant l'obscène et le trash, ce qui irradie l'ensemble de l'œuvre de Boyle demeure l'inquiétante étrangeté au service d'un discours féministe.

La femme qui hante l'œuvre de Boyle n'est pas à l'abri de transformations (essentiellement) animalières en vue de nouvelles créations monstrueuses, visant à remettre à chaque fois en scène la place et le rôle de la femme face à l'homme, face à elle-même, face à la famille. La société, quant à elle, est courtcircuitée au profit de l'individu comme représentant de son espèce, de Our Ancestor's Concern à Haunt en passant par Maypole, où les têtes de femmes en porcelaine blanche incarnent LA Femme, au-delà du jeu des coiffures et des traits qui les singularisent de près. En fait, Maypole s'inscrit dans une démarche de longue haleine où des motifs récurrents inscrivent le féminin dans son générique (l'escarpin doré, les galbes tous identiques) tout en créant un univers fantasmatique logique et cohérent dont l'artiste parle aussi volontiers que Redon et dans le même esprit: "J'éprouve vraiment le besoin de créer un autre monde, une vision de ce qui pourrait être magique et

\footnotetext{
9 Des sculptures de porcelaine d'Annie Attridge, on pensera à $A$ love that cannot forsake, Eye Spy, Tree Heart ou Delight my fucking Angel, qui reprend de manière plus brouillonne le motif des jambes en l'air qui sortent d'un socle dentelé de Boyle.
} 
magnifique et fantastique dans le fait d'être humain. ${ }^{10}$ ». C'est à ce titre que Maypole est un prolongement du curieux Little Brown Bat (2008), d'où cinq jambes sortent d'un jupon de dentelle chair et où l'on retrouve le motif de l'escarpin doré et celui de l'arbre, non comme mât cette fois, mais comme tronc coupé d'où sort le jupon. Écrasée par le jupon, une chauvesouris gît, laissant le spectateur perplexe, tiraillé entre ce qu'il sait (la «petite chauve-souris brune » ou vespertilion brun est la chauve-souris la plus répandue au Québec) et ce qu'il voit : elle est blanche, dans un environnement rose pâle.

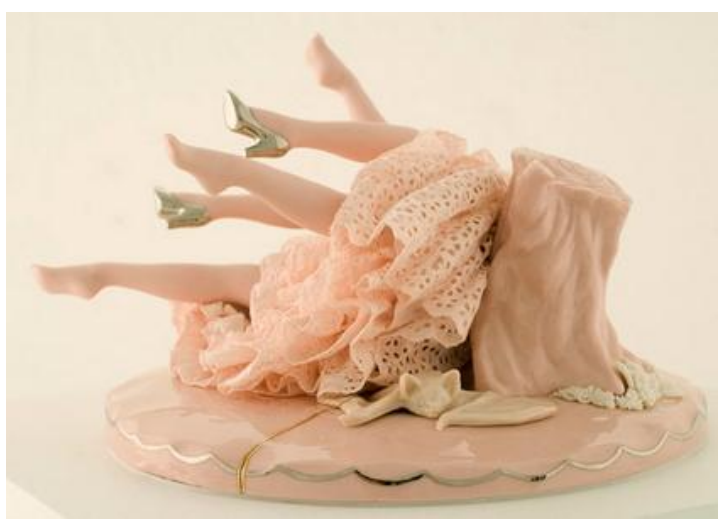

Little Brown Bat, Shary Boyle, 2008

Little Brown Bat fait partie d'un ensemble de figurines de porcelaine exposées à The Cave par la Galerie Jessica Bradley Art + Projects à Toronto ${ }^{11}$ en 2009. Elles ont particulièrement fait parler

\footnotetext{
10 Propos de Boyle rapportés par Heiti (2008, p. 64) : «I really feel the need to create an alternative, a vision of what might be magical and beautiful and fantastic about being human. ». (C'est moi qui traduis.)

11 Cette galerie représente l'artiste. L'exposition à laquelle je fais référence s'est tenue en février/mars 2009. Elle proposait une série de pastels et des figurines de porcelaines.
} 
d'elles, à partir d'un dénominateur commun qu'est le chiroptère. Celui-ci n'est pas sorti d'un roman gothique, même si sa présence y fait inévitablement penser et que nombre de compositions y font d'évidents clins d'œil. La raison est à la fois politique et écologique : depuis l'hiver 2006-2007, en Amérique du Nord, les spécialistes constatent, impuissants, la décimation de colonies de chauve-souris entières, par la faute d'un champignon dévastateur. Mettre en corrélation ces chauves-souris et les femmes ne relève donc pas d'un motif esthétique ou d'un clin d'œil à la fiction anglaise du $19^{\mathrm{e}}$ siècle. C'est de mort qu'il est question, d'enjeu international. On saisira pourquoi les chiroptères sont blancs en se reportant à la blancheur du museau, signe d'infection chez les chauves-souris. Boyle n'a fait qu'uniformiser son aspect crémeux. La mort a fait son œuvre, la femme métonymisée en jambes roses est-elle en sursis? La figurine Suspiria (2009) ne semble pas optimiste sur la question : la femme-chiroptère est suspendue par les pieds à un tronc.

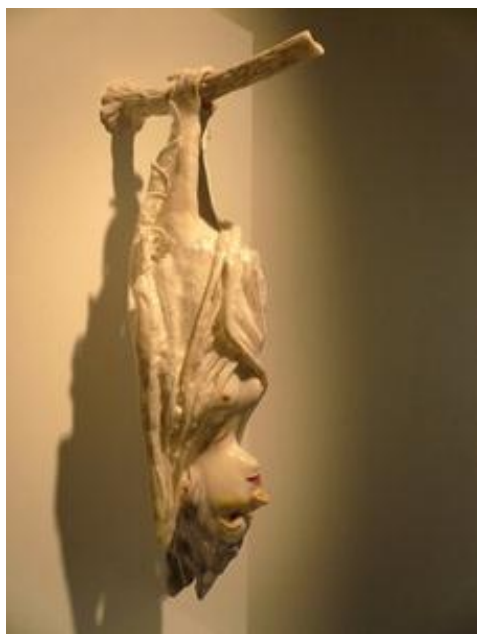

Suspiria, Shary Boyle, 2009 
Son visage est bien celui d'une femme, juste un peu étonnant avec sa langue coincée entre les dents, ses seins fermes le prouvent aussi, tandis que ses jambes cette fois n'ont rien du galbe rosé qu'on connaît si bien. Ce sont celles d'une bête poilue, renvoyant dès lors à Our Ancestor's Concern, présenté lors de la même exposition. Cependant, les poils ici sont tracés d'une main légère sur la porcelaine. Mais le chiroptère n'est-il pas un mammifère proche du primate? Une étrange parenté se forme, composée du singe, du chiroptère et de la femme, un drôle de compagnonnage dirait-on si la mort ne rôdait pas. Mais le trio, parce que sa violence symbolique est canalisée par la délicatesse du traitement de la porcelaine, interroge sans passer par le morbide ou la monstruosité terrifiante.

À l'instar de Redon, Boyle pourrait affirmer sans rougir : "Toute mon originalité consiste donc à faire vivre humainement des êtres invraisemblables selon les lois du vraisemblable, en mettant, autant que possible, la logique du visible au service de l'invisible. » Il y a cependant chez l'un et l'autre un certain nombre de conditions à cet exercice, dont le fait de ne pas entamer de course à la perfection technique, laquelle se ferait au détriment de l'imagination fertile en monstres tendres ou tristes. Ultime rapprochement: l'art de Redon était en marge de son époque par sa fantaisie à contrecourant du naturalisme; celui de Boyle aussi, qui refuse de déléguer comme beaucoup de ses collègues : «J'ai encore cette idée romantique et vieillotte que l'art a quelque chose à voir avec le spirituel », affirme l'artiste, précisant que, selon elle, « si l'on veut faire quelque chose qui ait un contenu spirituel 
parlant pour autrui, il faut toucher leur esprit, il faut s'y adresser physiquement ${ }^{12} »$. À cette condition, qui se traduit donc en maniant le fusain et en travaillant la porcelaine, les monstres jaillissent aux confins de l'esprit. Et de la technique qui les fait naître, ils gardent l'empreinte.

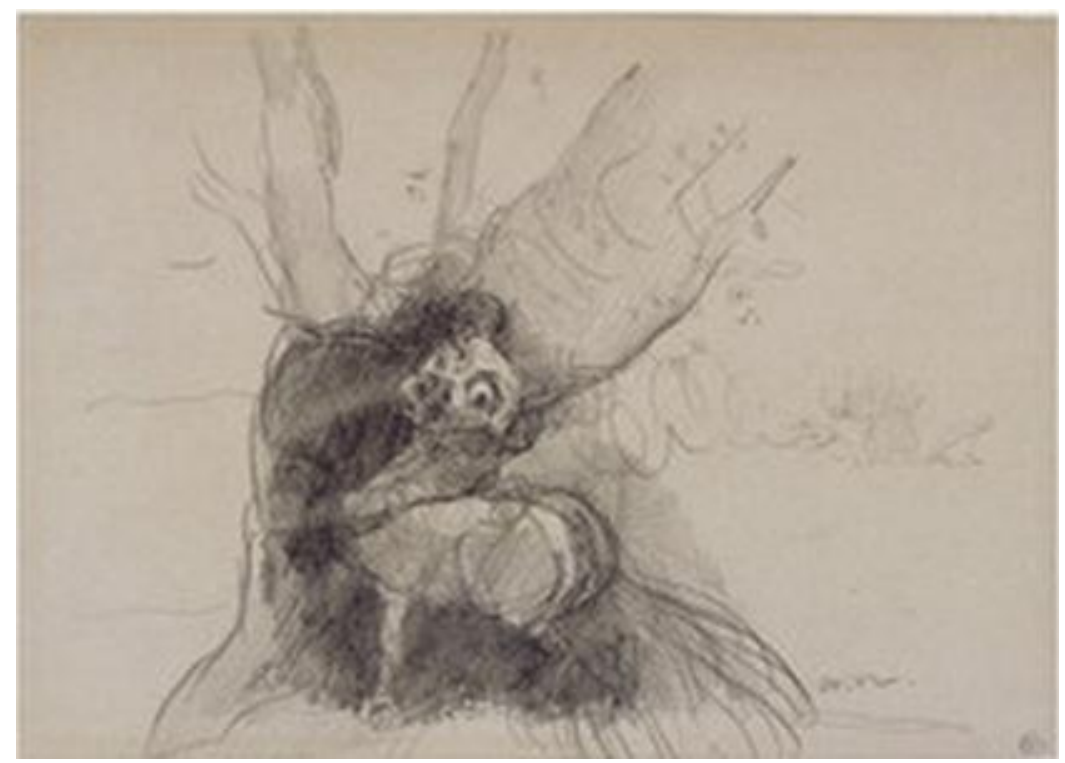

Petit monstre au pied d'un arbre, Odilon Redon

12 «I still have this old-fashioned romantic idea that art is something about the spirit [...] and I feel that if you want to make something that has a spirit, and speaks to the spirit of other people in the world, you have to touch it, you have to physically address it. » (propos rapportés par Everett-Green; traduction de Bertrand Rouby) 


\section{Bibliographie}

DESCARTES, René. (1637). Discours sur la méthode, Les passions de l'âme, Lettres, "Cinquième partie", Paris, Éditions du Monde Moderne.

EVERETT-GREEN, Robert. (2010), « The Hunger Artist », The Globe and Mail, September 14, p. 63.

HEITI, Sheila. (2008), Otherworld Uprising. Shary Boyle, Montréal, Conundrum Press, Lethbridge, Southern Alberta Art Gallery.

MACHADO, chevalier da Gama. (1831), Théorie des ressemblances, ou essai philosophique sur les moyens de déterminer les dispositions physiques et morales des animaux, d'après les analogies de formes, de robes et couleurs, Paris, Treuttel et Würtz, Delaunay.

REDON, Odilon. (1989), À soi-même. Journal 1867-1915. Notes sur la vie, l'art et les artistes, Paris, José Corti.

\section{Résumé}

Pour faire vivre les monstres peuplant son imaginaire sans les enfermer dans une représentation violente, l'artiste choisit des techniques et des matériaux qui infléchissent le sujet, tempèrent sa violence, éliminent les tensions. Ainsi, Odilon Redon compose ses créatures en clair-obscur, au fusain, avec la même inquiétante étrangeté que l'artiste ontarienne Shary Boyle, 150 ans après, 
obtient par la sensualité du pastel et la brillance d'une porcelaine traitée comme une dentelle. L'euphémisation, dans les deux cas, passe par l'esthétisation. L'apaisement, aussi, par une technicité qui accepte le spontané et voit en son imperfection le chemin vers l'humanisation du monstre.

\begin{abstract}
Whenever artists seek to bring to life their imaginary monsters without confining them within a violent representation, the techniques and materials they choose tend to deflect the subject matter, lessen their violent potential and alleviate tensions. Thus, Odilon Redon resorts to charcoal and chiaroscuro to sketch out his creatures, with the same uncanny overtones as in Ontarian artist Shary Boyle's sensuous pastels and lace-like lustrous porcelain. In both cases, the euphemization of violence is brought about by aestheticization. With their respective approaches, the two artists embrace spontaneity and humanise monstrosity by means of imperfection.
\end{abstract}

